\title{
Design and clinical evaluation of an image-guided surgical microscope with an integrated tracking system
}

\author{
Jaime Garcia Giraldez • Marco Caversaccio • Ion Pappas · Jens Kowal • \\ Urs Rohrer - Gaetan Marti • Charles Baur • Lutz-Peter Nolte • \\ Miguel A. Gonzalez Ballester
}

Received: 19 September 2006 / Accepted: 24 November 2006 / Published online: 13 February 2007

(C) CARS 2007

\begin{abstract}
A new image-guided microscope system using augmented reality image overlays has been developed. With this system, CT cut-views and segmented objects such as tumors that have been previously extracted from preoperative tomographic images can be directly displayed as augmented reality overlays on the microscope image. The novelty of this design stems from the inclusion of a precise mini-tracker directly on the microscope. This device, which is rigidly mounted to the microscope, is used to track the movements of surgical tools and the patient. In addition to an accuracy gain, this setup offers improved ergonomics since it is much easier for the surgeon to keep an unobstructed line of sight to tracked objects. We describe the components of the system: microscope calibration, image registration, tracker assembly and registration, tool tracking, and augmented reality display. The accuracy of the system has been measured by validation on plastic skulls and cadaver heads, obtaining an overlay error of $0.7 \mathrm{~mm}$. In addition, a numerical simulation of the system has been done in order to complement the accuracy study, showing that the integration of the tracker onto the microscope could lead to an improvement of the accuracy to the order of $0.5 \mathrm{~mm}$. Finally, we describe our clinical experience
\end{abstract}

J. Garcia Giraldez $(\bowtie)$ I. Pappas · J. Kowal · U. Rohrer . L.-P. Nolte · M. A. Gonzalez Ballester

MEM Research Center, Institute for Surgical Technology and Biomechanics, University of Bern, Bern, Switzerland e-mail: jaime.garcia@memcenter.unibe.ch

M. Caversaccio

Department of Head and Neck Surgery, Inselspital, Bern, Switzerland

G. Marti $\cdot$ C. Baur

IPR/LSRO2/VRAI Group, EPFL, Lausanne, Switzerland using the system in the operation room, where three operations have been performed to date.

Keywords Computer assisted surgery · Augmented reality $\cdot$ Surgical microscopy $\cdot$ Tracking $\cdot$ Clinical validation

\section{Introduction}

Image-guided, computer-assisted surgery has emerged to improve localization and targeting, to provide a better anatomic definition of the surgical field, and to decrease invasiveness. Usually, in image-guided surgery, a computer displays the surgical field in a CT/MR environment, using axial, coronal or sagittal views, or even a $3 \mathrm{D}$ representation of the patient. Such a system forces the surgeon to look away from the surgical scene to the computer screen.

An augmented reality system should enable surgeons to view hidden critical structures such as pathologies (e.g. tumors), risk regions or sensitive structures (e.g. arteries or nerves), or the results of a preoperative planning, such as pathways, trajectories or distances. These data should be shown as if they were beneath the surface of the surgical scene.

Different techniques are applied to this end, for example navigation systems $[4,8]$ where surgical data and guidance are shown. In addition, overlay systems have been developed in order to display the surgical images directly to the patient. Semi-transparent screens can be used to display the images between the patient and the surgeon $[6,13,16]$. Stereoscopic binoculars are also used in augmented reality systems $[3,11,15]$. They provide surgical guidance including $3 \mathrm{D}$ perception, but they 
require the surgeon to wear a cumbersome headmounted device.

Surgical microscopy is used in many complex procedures in the area of otorhinolaryngology and neurosurgery. To avoid the need to look away from the surgical scene, guidance information can be overlayed directly on the surgical microscope view. In order to display a correct overlay image, changes in patient position have to be determined. Several approaches have been developed, mainly using external tracking systems [1,5, 12]. In this case, the motion of both patient and microscope need to be tracked. Alternatively, to circumvent the need for optical tracking, systems based on integrated position sensors [19] or image processing have also been developed [9].

We have developed an image-guided microscope with a new design concept, which consists in mounting a small and precise 3D tracking camera directly in the surgical microscope. The camera tracks the movements of the surgical tools and of the patient. This setup, which is described in more detail in the following section, offers improved ergonomics and a gain in accuracy compared with externally tracked systems. In this paper we describe the procedure of the integration of the tracking system in the microscope, which includes the calibration of the microscope optics and the registration of the tracking camera to the microscope. We propose a pair-point and a surface matching method to register the patient by using a marker attached to a dental splint, and we describe the different overlay visualizations the system can provide.

A complete accuracy study is then described in order to validate the concept by measuring the error of the overlay in different environments, starting with phantom skulls, cadaver studies, a precise computer simulation of the augmented reality system and finally several clinical cases performed in the operating room. As explained in Sect. 5, the system has been successfully used three times in Ear-Nose-Throat surgery(ENT).

\section{Materials and methods}

\section{System description}

The image-guided microscope consists of a Leica M500 surgical microscope with a Leica DI C500 image injection module, which allows the injection of color overlay images in one of the eyepieces. With this microscope the surgeon has the option to change the zoom and working distance during the operation, so these parameters should be modelled by our system. The surgeon has also the possibility to modify the brightness and the opacity of the overlay. The microscope is balanced in order to be able to move it smoothly, so it is very important to take this into account when adding any extra weight. We chose a small active optical tracking camera, the easyTrack 200 (Atracsys), which offers a tracking accuracy of $0.2 \mathrm{~mm}$ (Fig. 1) with a working volume that fits perfectly with the area below the microscope (Fig. 2). To track the movements of the patient, a maxillary splint with a mounted infrared marker-shield is used. Marker shields are also mounted on the surgical tools to track their motion. The software modules composing the application are reusable and plug into a software framework dedicated to computer-assisted surgery (CAS) applications.

To correctly inject scaled and rendered images as augmented reality overlays, the following information is needed (see Fig. 3): microscope calibration, registration of the microscope to the tracker and registration of the patient to the pre-operatively acquired image data.

\section{Microscope calibration}

Camera calibration is an indispensable step in any machine vision system. Among the different calibration techniques, the photometric calibration method is currently the most widely used in the medical field [17, 20]. It consists of observing a calibration object, like a chessboard or a planar grid, whose geometry in the $3 \mathrm{D}$ space is known with a good level of precision. This technique uses a snapshot representing the calibration object at different poses and extracts the object's features in the image, to finally correlate them with the $3 \mathrm{D}$

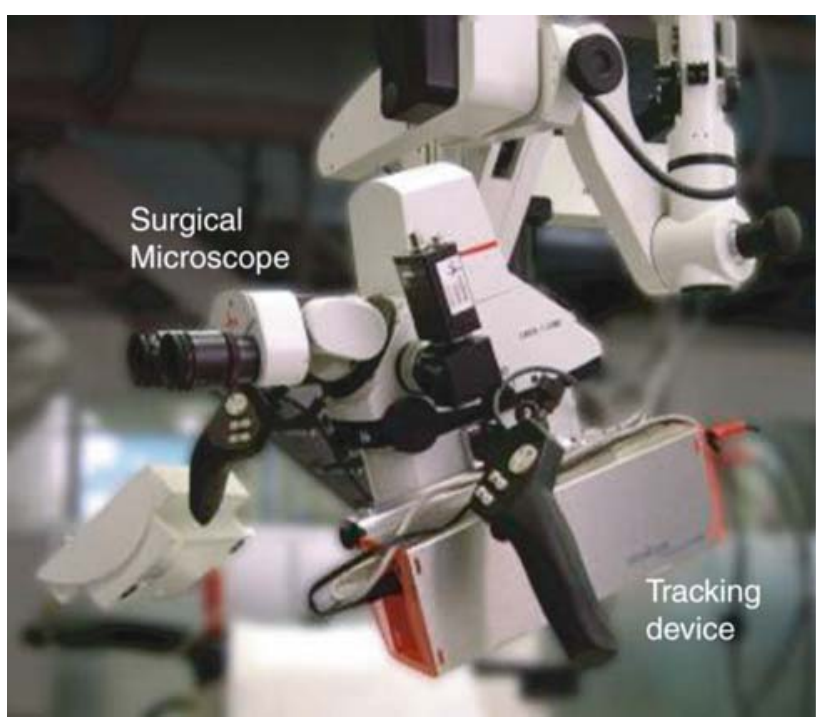

Fig. 1 The surgical microscope with the integrated tracking device 


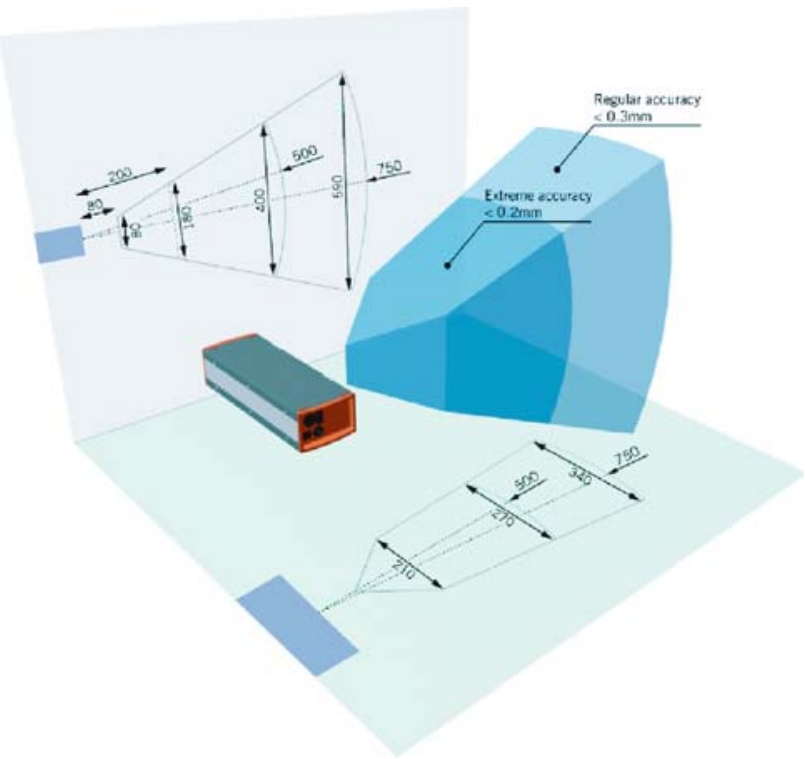

Fig. 2 Working volume of the easyTrack 200 (courtesy of Atracsys Sarl.). The size of the camera is $288 \mathrm{~mm} \times 98 \mathrm{~mm} \times$ $65 \mathrm{~mm}$, with a weight of $1.2 \mathrm{~kg}$

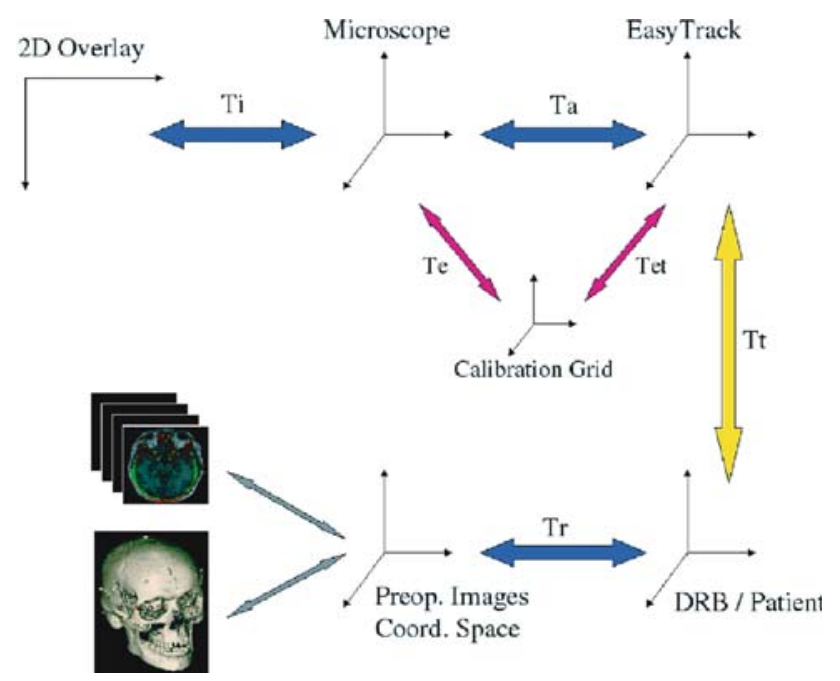

Fig. 3 System diagram: coordinate transformations involved in the overlay generation

model of the reference object. A calibration algorithm then extracts the homography matrix and the camera's intrinsic parameters (focal length, optical center, skew and radial distortion), as well as the extrinsic parameters (rotation and translation of the calibration object relative to its model coordinates).

Calibration of the optics of the microscope is performed using the method of Heikkila [10] with a pinhole model for $3 \mathrm{D}$ to $2 \mathrm{D}$ projection, implemented in the camera calibration toolbox for matlab by Bouguet. The extrinsic parameters $T_{\mathrm{e}}$ consist of the $3 \mathrm{D}$ translation and rotation vectors that bring the object frame of reference into the frame of reference of the microscope optics. The intrinsic parameters $T_{\mathrm{i}}$ include focal length, principal point, and radial and tangential distortions.

Due to the optics of the surgical microscope, with very high focal lengths, the effects of the distortion parameters are of the order of less than $1 \%$. Thus, those parameters can be removed from the model, simplifying the computation during the generation of the overlay images.

This model is based on a full perspective projection. Series of images are taken at different positions and angles of a 2D calibration object to successfully determine the necessary extrinsic and intrinsic parameters. We have used a planar grid as a 2D calibration object, obtaining an accuracy of 0.5 pixels rms. The error in the calibration of the microscope optics is a combination of the error in the estimation of the intrinsic and extrinsic calibration parameters, and of the error inherent to the calibration standard. This error is evaluated by comparing the pixel distance between all the grid points extracted from an image, and the points projected using the calibration parameters.

Several calibrations at different zoom levels and working distances are performed in order to create a lookup table. The surgeon will have the possibility to switch between them during the operation. Development of an optic model of the microscope is currently in progress to be able to use any zoom and focus.

\section{Patient registration}

In order to be able to navigate through the preoperative images, such images have to be registered to the patient coordinate system. To this end, a dynamic reference base (DRB) made with an infrared marker-shield is fixed to the patient with a dental splint (Fig. 4a). This DRB is tracked so the system constantly knows the relative position between the patient and the microscope. Two registration methods have been implemented in our system: pair-point matching and surface matching.

Pair-point matching consists in identifying corresponding points from preoperative images in the patient. Such points can be either fiducial markers screwed on the patient [14] or some anatomical landmarks (Fig. 4b), typically two points from both frontal processes, one point from the nasal bone and the last one in the anterior nasal spine. The second option has been retained since it is less invasive and accurate enough (below $1 \mathrm{~mm} \mathrm{rms}$ in the accuracy studies performed in laboratory). The registration matrix $T_{\mathrm{r}}$ is calculated by digitizing the chosen points using a tracked needle-pointer and applying a least-squares fitting algorithm [18]. 


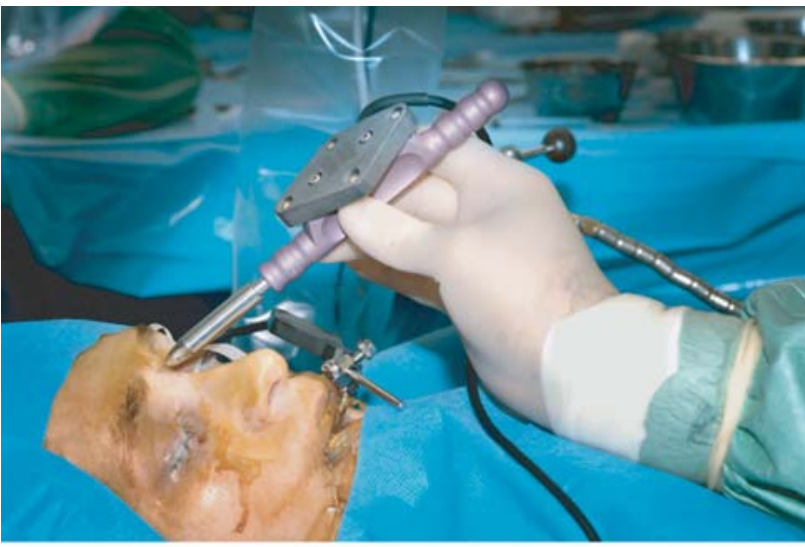

(a) The DRB is fixed with a dental splint to the patient. The surgeon digitizes the selected points with a tracked pointer.

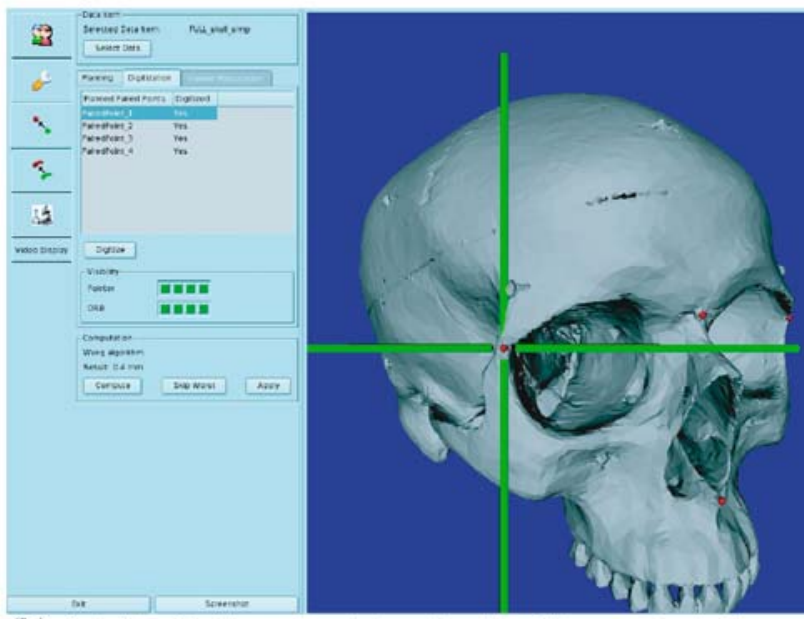

(b) Interface for the pair-point registration. Four anatomical landmarks are selected in a 3D model of the skull.

Fig. 4 Registration of the patient to the pre-operative image

Surface matching can be used after a pair-point registration in order to increase its accuracy. The surgeon uses the tracked needle pointer to digitize more points from the skull surface of the patient. Those points can be reached through the skin, so we do not need to expose the bone. However, in order to prevent from bending the needle, the surgeon has to proceed carefully. An iterative closed point (ICP) based algorithm [2] is applied to geometrically align the cloud of points to a 3D model of the patient previously segmented from the pre operative images. About 50 points from the frontal, zygomatic and maxilla bones have been digitized in every clinical test improving the pair-point registration to $0.6 \mathrm{~mm}$.

It is now widely accepted that the error metric that should be used is target registration error, TRE [7], that means calculating the error at points other than those used for registration and not simply quoting an RMS residual. Regarding the pair-point matching procedure, after every registration up to 20 more points are digitized in the region of interest with a tracked pointer. In order to have a prediction of the TRE, the accuracy of the registration is then calculated measuring the mean distances from those points to the surface. In the case of surface matching, the cloud of digitized points is used to build a 3D surface, which is then compared to a surface model of the digitized region, build from CT data. Thus, the error calculated is the mean distance between both surfaces.

The registration is verified several times during the operation because of possible movements of the dental splint. To do so, the surgeon uses a tracked pointer and a CT viewer where he can verify the position of the tool. Thus, in case of movement we go back to the registration procedure.

\section{Tracker-microscope integration}

The novelty of our design is the integration of the tracking system to the microscope. In doing this, several clinical and technical constraints need to be met. Two main aspects have been taken into account: ergonomics in the OR and balancing of the microscope.

Most existing navigation systems require the installation of several devices in the operation room (OR), e.g. tracking cameras and workstations. However, the working space during an operation is limited, and the number of people and surgical tools participating in the operations have increased in recent years. Therefore, the ergonomics of the system becomes an important issue. To this end, the tracking system has been integrated in the microscope in order not to occupy resources, and depending on the available space in the OR the system can be installed either in a desktop computer or on a laptop.

Moreover, since a surgical microscope is a highprecision system used in clinical interventions, the addition of the tracker to the microscope should not disturb in any case the clinical procedure. Several parameters have been taken into account in the design of the tracker fixation. First of all, the working volume of the tracker is very important to locate the tracker in the microscope, and it should fit with the working distance of the microscope during any operation. However, the working distance changes depending on the kind of operation, so the fixation should be adaptable. Secondly, the Leica M500 microscope offers an advanced movement system to achieve balance in six axes and all locations/angles of the surgical microscope, so the weight and position of the tracker should maintain correct operation of the auto-balance system.

Depending on the operation, two configurations have been designed. The first one is a lateral fixation, with an 
angle of view that was quasi-parallel to the optical axis of the microscope (Fig. 5b). With this setup the field of view of the microscope remains always inside the working volume of the tracker. However, in some operations the distance from the microscope to the patient is not enough to guarantee a correct visibility of the tracked

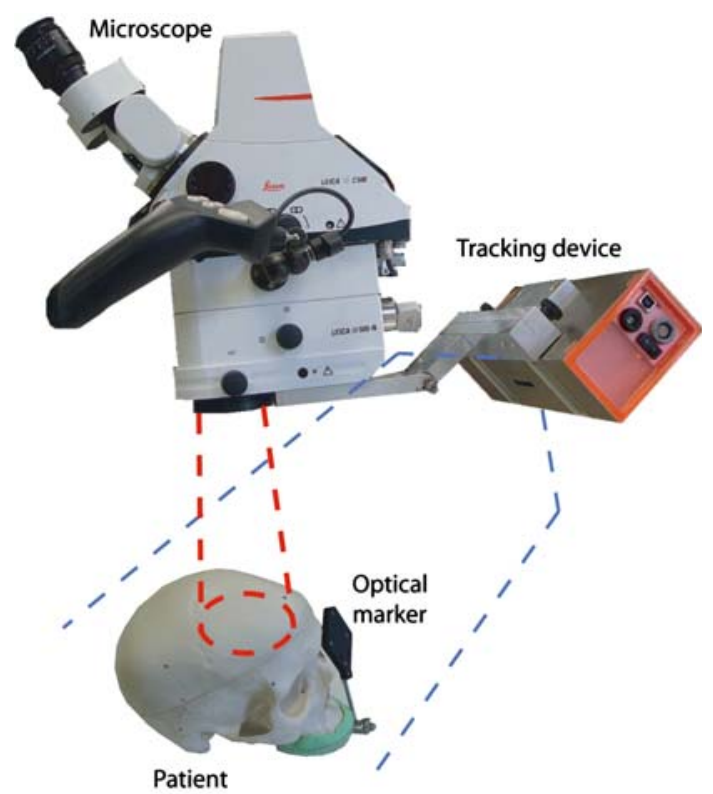

(a) Posterior fixation

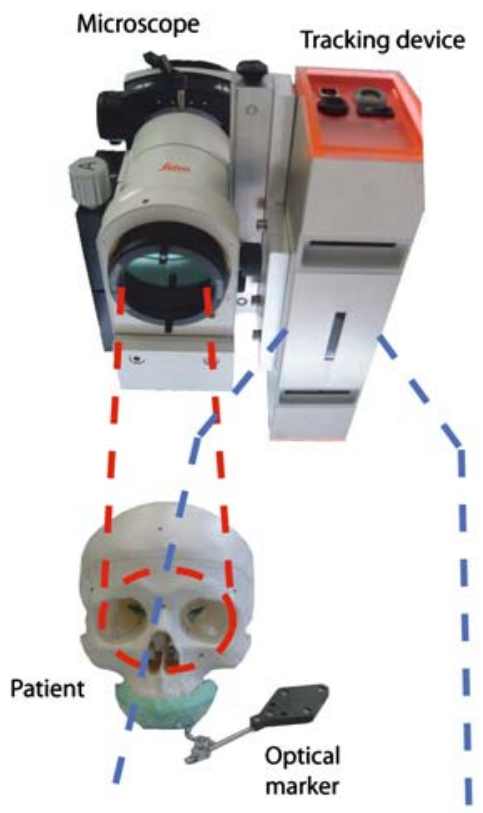

(b) Lateral fixation

Fig. 5 Two designs for the tracking integration. The position of the tracking camera can be changed depending on the working distance needed for the operation and the position of the patient. The dashed blue line represents the working volume of the tracking device, and the red line the field of view of the microscope tools. To address this problem, the second design is a posterior fixation (Fig. 5a), increasing the working distance. In addition, we provide a mechanism to adapt the angle of the tracker axis with respect to the optical axis of the microscope. However, all the changes in the fixation have to be made always before to enter to the operation room due to the registration and calibration of the microscope and tracker (Sect. 2.5).

Regarding the balancing of the microscope, two parameters are to be taken into account: the weight of both tracker and fixation and their position. The influence of the position of the tracker in the microscope is solved by using a counterweight in the opposite side. However, the head of the microscope is carried by a controlled hydraulic arm with a limited strength. The surgical microscope used in the operation room is already equipped with an spy viewer and a video camera, so being $1.5 \mathrm{~kg}$ the weight of the tracker, the weight of the fixation has been minimized to $0.5 \mathrm{~kg}$ to avoid problems of balancing due to overweight. The total addition of weight of our system rises to $3.5 \mathrm{~kg}$, being perfectly supported by the balancing system.

\section{Tracker to microscope registration}

Due to the working distance, typically from 30 to $60 \mathrm{~cm}$, small errors in the calibration and registration of the tracker are magnified in the region of the patient, so only an accurate procedure will validate the concept.

The registration of the tracker to the microscope is needed to have a transformation between the microscope coordinate space and tracking space ( $T_{a}$ in Fig. 3). To this end, a tracked marker is fixed to the plate containing the standard grid used during the calibration (Fig. 6). Several points from the grid are then registered to the marker and defined as imaginary points. Those imaginary points are defined following the same coordinate system as used in the optics calibration. The digitization of the imaginary points is made by using a master pointer with $0.1 \mathrm{~mm}$ of accuracy.

At the same time as the optics calibration procedure, the acquisition of the images is synchronized with the acquisition of the tracked positions of the imaginary points. Transformations $T_{\mathrm{e}}$ with the extrinsic parameters and $T_{\mathrm{e}} t$ with the imaginary points are then calculated for every single frame, obtaining as much $T_{\mathrm{a}}$ as images acquired. Finally we calculate $T_{\mathrm{a}}$ by applying a least squared method with all $T_{\mathrm{e}}$ and $T_{\mathrm{e}} t$, thus minimizing the error.

Therefore, any position in the tracker coordinate system $\left(\vec{X}_{\mathrm{t}}\right)$ can be expressed in the microscope coordinate system $\left(\overrightarrow{X_{\mathrm{m}}}\right)$ as follows: 


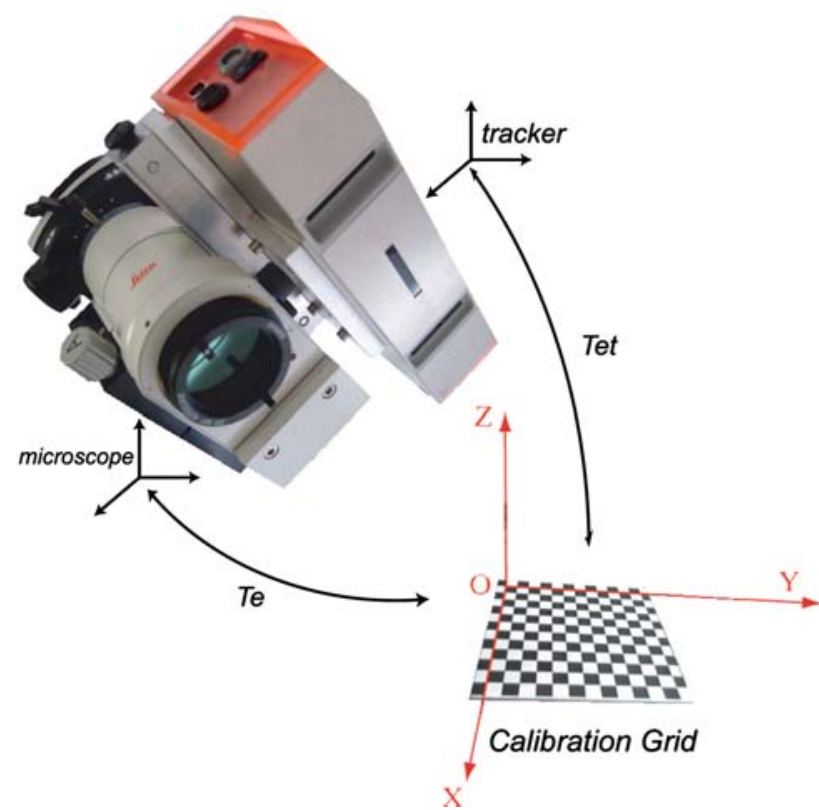

Fig. 6 Transformations involved in the registration of the tracker to the microscope

$\overrightarrow{X_{\mathrm{m}}}=T_{\mathrm{a}} \cdot \vec{X}_{\mathrm{t}}$

where $T_{\mathrm{a}}=T_{\mathrm{e}} \cdot T_{\mathrm{et}}$.

This registration is always performed after the final positioning of the tracker on the microscope, that means choosing the lateral or posterior position and the angle. Since the tracker is fixed, it can not be moved during the time of the operation.

The total error of the registration is $0.3 \mathrm{~mm}$. That takes in account the error of the calibration and the error of the digitization and tracking of the grid. However, the goal of this project is the integration of the tracking system with the microscope, so as shown in Sect. 4, the accuracy of the overlay could be significantly improved by reducing the error of the registration of the assembly. That could be made possible by optimizing the physical integration of the tracker in the microscope, building a robust fixation of very accurately known geometry. The calibration procedure could also use robotized positioning of the grid in order to improve the $0.2 \mathrm{~mm}$ accuracy of the tracking system.

\section{Augmented reality overlay}

Although 3D visualization is the goal of most of the existing augmented reality systems, it is hard to achieve a good depth perception. Depending on the user, displayed structures can be perceived above or below the skin. Several visualization techniques are applied to be easily perceived by a larger majority of people [5]. Instead of trying to solve this problem, our efforts have been concentrated in developing a monocular overlay system independent from the user perception that provides useful information to the surgeon. The 3D perception is replaced by interactive tools to be guided and to measure distances and trajectories. In addition, several modalities of overlay have been developed, including a $\mathrm{CT}$ viewer where the surgeon can choose a plane from the CT and display it as an overlay in the patient, and a $3 \mathrm{D}$ viewer to render $3 \mathrm{D}$ models of segmented structures.

Moreover, the system is implemented using a commercial overlay system provided with the surgical microscope Leica (Leica DI C500), so it is not needed to incorporate any extra hardware, making the final system less expensive.

\section{CT viewer}

Usually, in image-guided surgery, a computer displays the surgical field in a CT/MR environment, using axial, coronal or sagittal views. The goal of our CT viewer is to avoid the need to look away from the surgical scene, as is required in existing CAS systems. Thus, once the patient has been registered the system tracks its position and displays correctly scaled CT cut-views through the image injection module to the microscope view (Fig. 7a). The cut-view of the CT will correspond either to a plane orthogonal to the optical axis of the microscope, selected with a tracked pointer, or containing the direction of the pointer and parallel to the microscope plane in order to see its position inside the patient.

\section{$3 \mathrm{D}$ viewer}

Using the data from the calibration of the microscope and the tracking coordinates we can create a virtual camera, which will render the scene to the overlay (Fig. 7b). Before the operation, preoperative planning has to be done in order to create the 3D models needed from the CT or MRI datasets. Such models can be, depending on the operation, segmented tumors or targets, risk regions like nerves or arteries, or access pathways to guide the surgeon. Although the system only works with one of the eyepieces, so the surgeon does not have a depth perception, the perception can be replaced by using virtual tools with distance measurements and trajectories, as described next.

\section{Tracked tools}

A typical problem in augmented reality systems is that, once the overlay is activated, the tools used by the 
Fig. 7 Screenshots from the microscope viewer during cadaver experiments

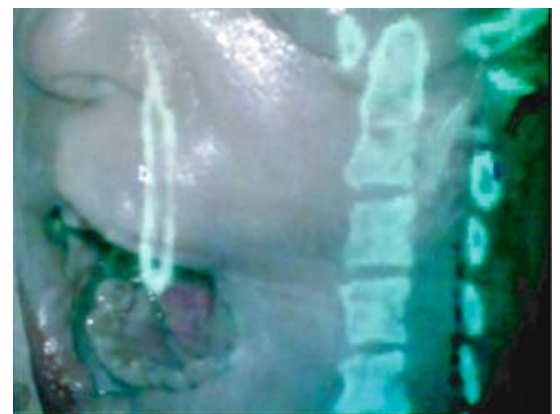

(a) CT plane displayed in microscope viewer

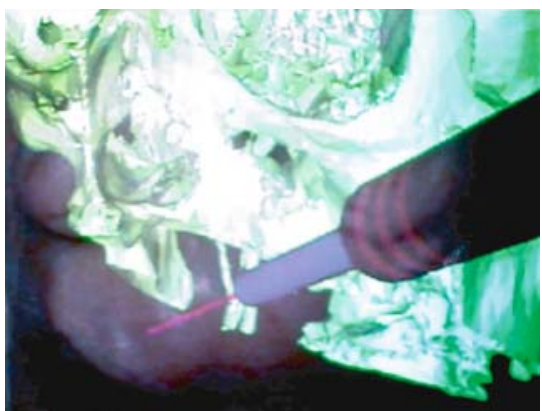

(b) Display of a 3D model of the skull

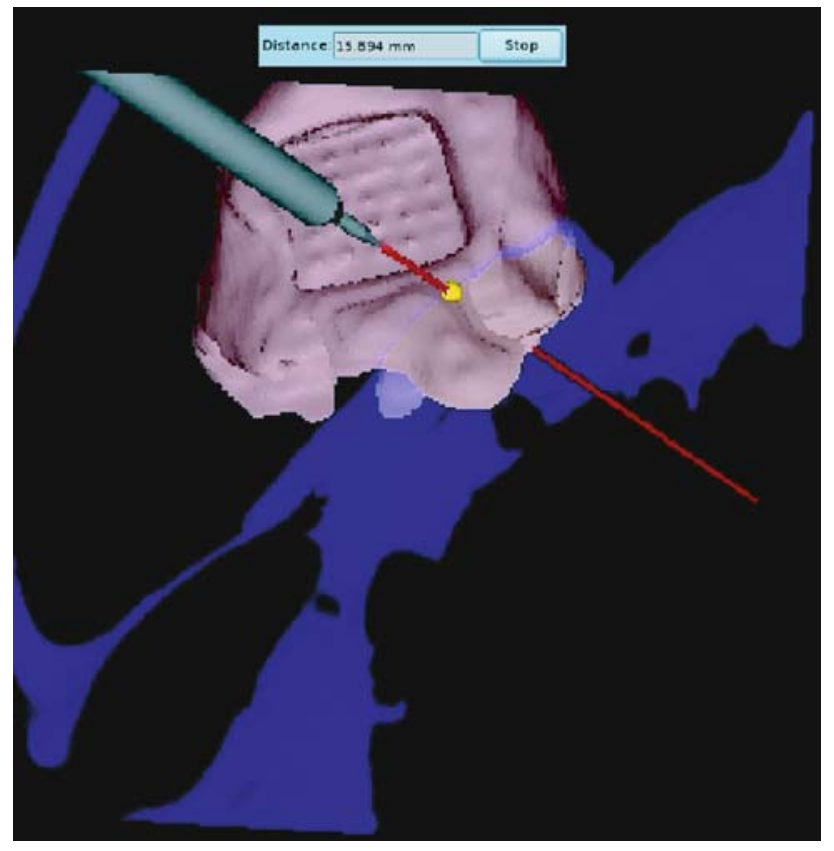

Fig. 8 Image projected to the microscope: 3D model of a simulated tumor(red) and a CT cut-view (blue). The model of the tool is shown, then the trajectory is projected, calculating the intersections with the targets and measuring distances

surgeon appear below it. To avoid this problem, since the tools can be tracked, 3D models of them can be projected in the overlay as a virtual tools so the surgeon can see their position (Fig. 8). Additionally, when using the tracked tools, the system can project the current trajectory of the tool, together with distance measurements and intersections. This concept increases the interactivity of the surgeon with the guidance system, as well as giving some depth information to the augmented reality overlay.

\section{Accuracy study}

As a precursor for clinical tests, a study of the accuracy of the augmented reality system was performed. Since the goal of the system is to guide the surgeon during the operation, by showing targets and risk regions, the accuracy of the overlay image should be enough in order to prevent any damage. Typically in neurosurgery and ENT interventions the upper limit of the error is related to the size of the tools, i.e. 3 or $4 \mathrm{~mm}$ when using pointers or needles. Since the new imaging systems like CT or MR can provide sub-millimeter accuracy, the goal of our system should be a millimeter accuracy. However, this accuracy should be better when approaching critical regions, i.e. nerves.

In addition to the evaluation of the registration, explained in Sect. 2.3, the virtual tracked pointer has been used during the study in order to measure distances and to have a visual inspection of the projected overlay.

\section{Grid and phantom study}

In order to have a first evaluation of the accuracy of the overlay a grid of metallic balls has been used as a testing object. The metallic balls, of $1 \mathrm{~mm}$ of diameter, are arranged in two grids of 25 balls in two $z$-levels (Fig. 9a), separated $4 \mathrm{~mm}$ in $X$ and $Y$, and only $5 \mathrm{~mm}$ in $Z$ due to the small depth of field at the highest zoom $(1 \mathrm{~cm})$. A fixed marker shield has been fixed to the plate containing the grid, and a CT of the plate was then registered using a pair-point matching of 8 points from the grid with $0.2 \mathrm{~mm}$ accuracy. The accuracy of the overlay is calculated capturing images from the eyepiece of the microscope with and without the CT overlay displayed (Fig. 9b). Centers of the balls in both real and overlay images are segmented. The pixel to millimeter ratio is calculated by using the size of the grid. Finally the overall overlay accuracy has been estimated by measuring the distances between the centers in $\mathrm{mm}$, with a mean result $0.4 \mathrm{~mm}$ in $X$ and $Y$ axis. The accuracy in $Z$ was measured by moving the grid test up and down with a micrometer table. 
Fig. 9 First evaluation of the accuracy of the overlay system using a custom-made validation grid and a plastic skull phantom

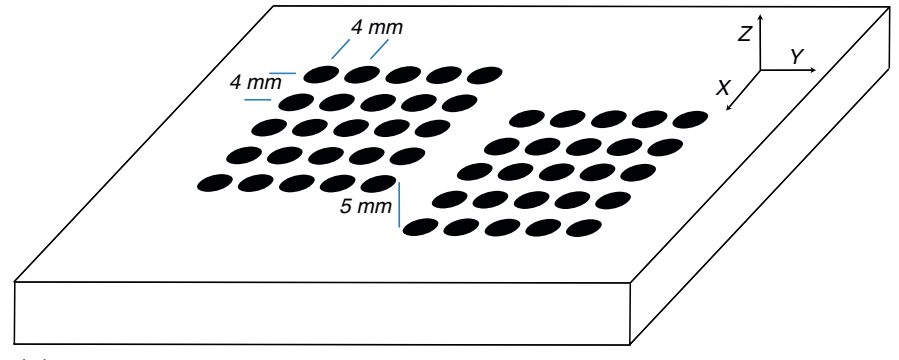

(a) Schema of the grid containing the metallic balls.

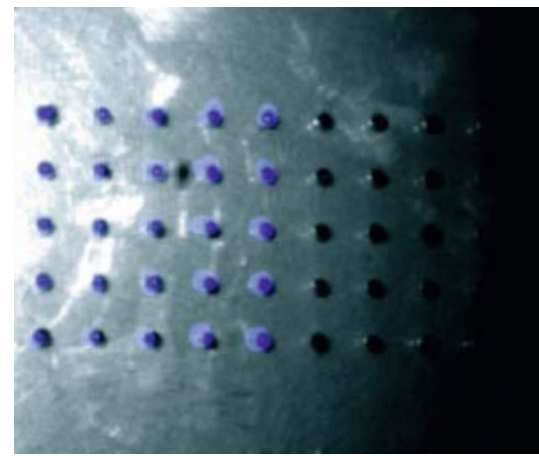

(b) Metallic grid view

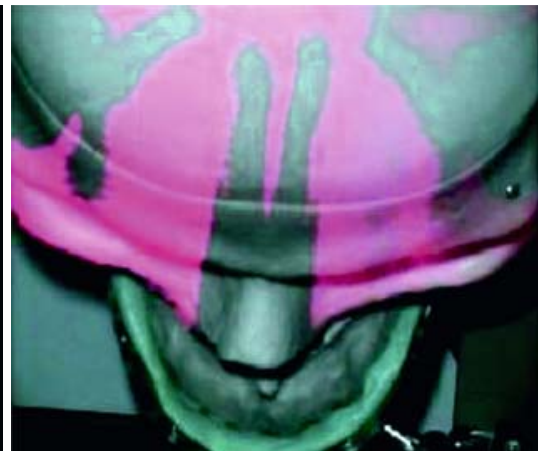

(c) Phantom skull view
A second evaluation was performed to assess the effects of the registration in the overlay generation. A plastic skull was equipped with four fiducials and a marker shield was fixed to it using a dental splint. The error of the registration in this case was $0.3 \mathrm{~mm}$, instead of the $0.2 \mathrm{~mm}$ obtained with the grid. The accuracy of the registration when using anatomical landmarks went from 0.3 to $0.4 \mathrm{~mm}$. As shown in Fig. 9c, a CT cut-view was then projected to the overlay. In this case the accuracy was calculated by looking at the difference in pixels of several contours in both real and overlay image, obtaining a mean of 3-4 pixels that corresponds to an accuracy from 0.5 to $0.6 \mathrm{~mm}$ (pixel to millimeter ratio calculated previously).

All the measurements where taken inside a region of interest with a radius of $10 \mathrm{~cm}$ from the marker shield. As explained in Sect. 4.3, the error increases with the distance from the marker.

\section{Cadaver experiments}

Validation on cadaver heads have been done in order to check more aspects of the system, like the registration procedure and the visualization.

In these experiments, selected anatomical landmarks on the skull were used for the registration. Though the access to the rigid parts was more difficult than in phantom skulls, we obtained an accuracy of $0.6 \mathrm{~mm}$ after a surface matching registration.
When using a cadaver head it becomes obvious that the visualization is the critical aspect of an augmented reality system. As the surgical scene can contain many different colors (blood, tissues...), the surgeon should have the possibility to modify some parameters from the overlay like colors, contrast or brightness. To this end, our system provides a menu that is accessible during the surgical intervention.

Finally we validated the overlay (Fig. 7) by measuring its accuracy at the following anatomical landmarks of the skull: supraorbital notch, infraorbital foramen, frontozygomatic suture, anterior nasal spine, nasal bone and external acoustic meatus. The measured overlay accuracy was $0.8 \mathrm{~mm}$, with only $0.7 \mathrm{~mm}$ when we were close to the DRB position and $1.5 \mathrm{~mm}$ at the farthest position. As explained in the previous section, the accuracy was measured looking at the contours. To this end we cut the skin and we exposed the regions of interest.

\section{Accuracy simulation}

In order to complement these studies a numerical simulation of the overlay generation has been done. Four steps are simulated in the system (see Fig. 3): calibration of the microscope $T_{\mathrm{i}}$, registration of tracker-microscope $T_{\mathrm{a}}$, tracking $T_{\mathrm{t}}$ and patient registration $T_{\mathrm{r}}$. For each step we measured the mean error values from the previous accuracy studies: 
1. Calibration: 0.5 pix.

2. Tracker-Microscope registration: $0.3 \mathrm{~mm}$.

3. Tracking: $0.2 \mathrm{~mm}$.

4. Patient registration: $0.6 \mathrm{~mm}$ (pair-point and surface matching).

Another important parameter is the distance from the microscope to the patient. As explained in Sect. 2.5, it has a great influence in the accuracy of the overlay. For the simulation, several measures of that distance have been taken from different kinds of surgical interventions, and a mean value of $35 \mathrm{~cm}$ has been retained. By taking a typical set of values for the transformations involved in the system, we have calculated the corresponding overlay-position $\overrightarrow{X_{p}}$ of 200 points $\overrightarrow{X_{w}}$ randomly selected from the patient volume:

$\overrightarrow{X_{p}}=T_{g} \cdot \overrightarrow{X w}$

$T_{g}=T_{i} \cdot T_{a} \cdot T_{t} \cdot T_{r}$

The errors, modelled as Gaussian noises, have been included in the different stages of the overlay generation to obtain a new $T_{g}^{\prime}$. To measure the error in $\mathrm{mm}$, a backprojected line $L_{p}$ from the image position $\vec{X}_{p}$ is created, and the distance $d_{s}$ from the nearest point on this line to the corresponding point $\overrightarrow{X_{w}}$ in the patient volume is measured (Fig. 10).

Results of the simulation are shown in Fig. 11. After 5,000 iterations, we obtain a similar behavior as shown in the cadaver study, a similar range of error values and a strong dependency with the distance from the marker frame (solid line).

With this simulation we are able to validate the concept of the integration of the tracking system in the microscope. As shown in Fig. 11, the dashed line represents the accuracy of the overlay after removing the error due to the registration of the tracker to the microscope $T_{\mathrm{a}}$. Thus, this should be the maximum accuracy we could reach, so reducing the error in $T_{\mathrm{a}}$ will approach the system to this limit.

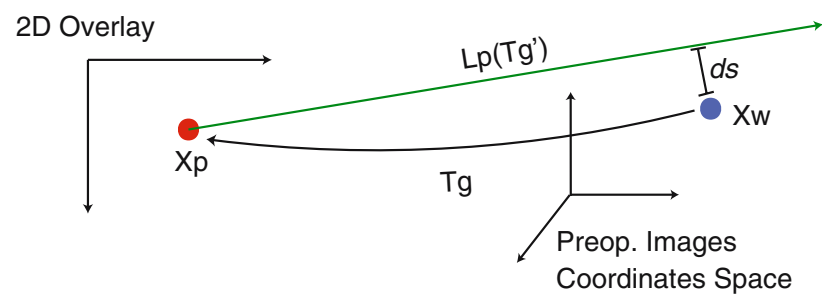

Fig. 10 Overlay accuracy quantification by backprojection

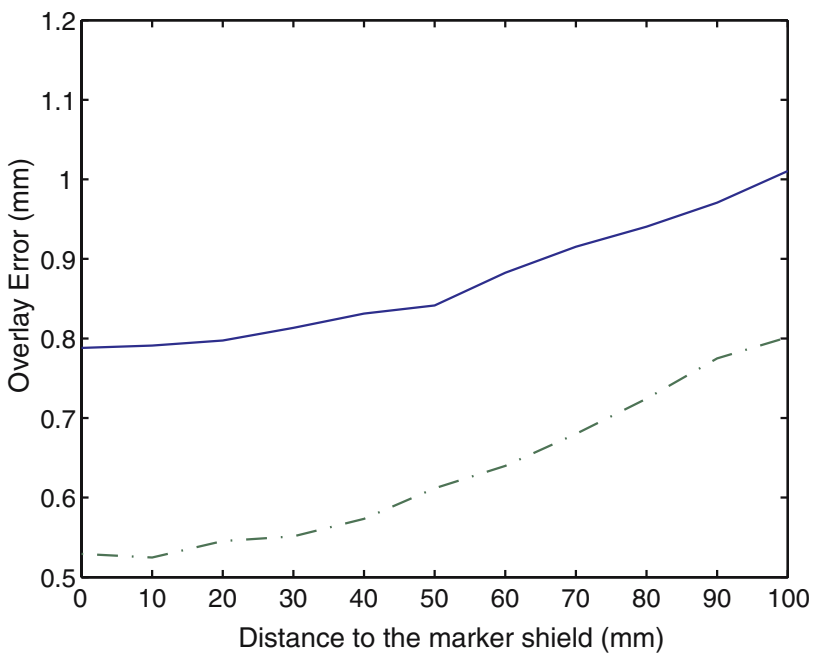

Fig. 11 Overlay error simulation: the error increases as a function of the distance to the marker (solid line). This error can be significantly reduced by integrating the tracker in the microscope (dashed line)

\section{Clinical evaluation}

\section{Operation room procedure}

The validation studies reported above prove that our system has a sub-millimeter accuracy, appropriate for clinical use. Several aspects are of importance to enable use of the system in the OR, such as sterilization and ergonomics.

A sterile environment must be ensured throughout the operation. Since the tracking camera is integrated in the microscope, the plastic bag that covers the microscope was adapted in order to also cover the tracker (Fig. 12). Three windows were made in the bag, corresponding to the three CCD, to prevent from any reflection error in the optical tracking.

As mentioned in Sect. 2.4, two configurations for the fixation of the tracker were created, in order to ensure optimal ergonomics and visibility of the tools and DRB. In our clinical experience, we found that the posterior fixation was more adapted in two of the cases. Moreover, the balance of the microscope was obtained more easily in this position.

Another important aspect regarding the ergonomics is the installation procedure. Since the free space and the time in the operation room is limited, the integration of the tracker in the microscope and its installation time becomes an important factor. For the cases reported, a day before the operation, the fixation of the tracker to the microscope and its balancing were achieved in about $15 \mathrm{~min}$, and the calibration procedure took $30 \mathrm{~min}$. In a final system, the fixation would be permanent, and 


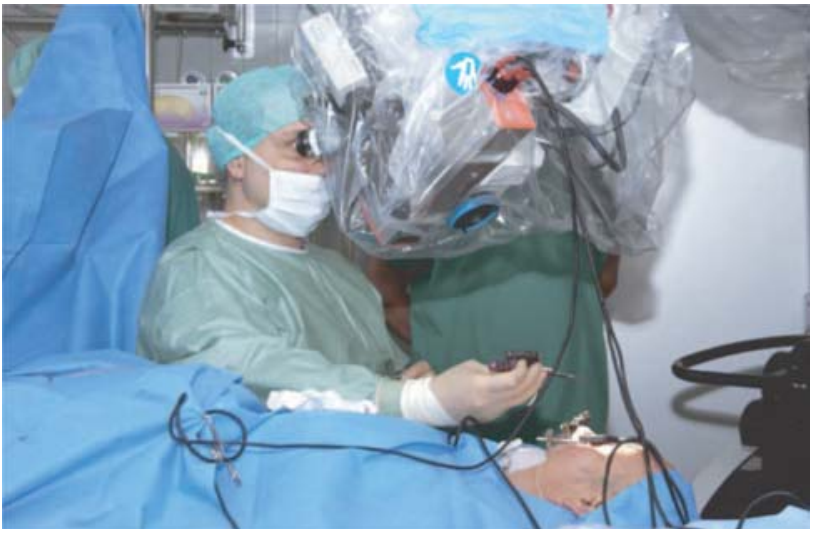

Fig. 12 Use of our image-guided microscope system in the operating room. The sterile bag used to cover the microscope also encloses the tracker, ensuring safety and optimized ergonomics

the calibration made very accurate (or even avoided by building a precise fixation of known anatomy). The fact of having the system installed on the microscope did not add any extra time to the normal OR preparation procedure before the operation, as the placement of the tools and cables and the software initialization was done in parallel to the standard preparations performed by the hospital staff, and calibration was done the day before.

\section{Clinical results}

To date, the system has been employed on three patients. The first two tests were performed with the tracker installed in the lateral position. Such position was changed to a posterior in the last test to have a better visibility of DRB and tools.

The first patient underwent removal of polyps in the right maxillary sinus and the second patient was affected of a mucocele in the right frontal sinus. The procedure was similar in both cases. The DRB was fixed to the patient with a dental splint, then the surgeon performed the registration obtaining 0.6 and $0.8 \mathrm{~mm}$, respectively. In both operations the surgeon was able to visualize CT cut-views and projections of 3D models of the targets previously segmented from the CT datasets. Those operations are performed normally with endoscope, so we did not have direct view to internal structures, making difficult to calculate the accuracy of the system. However, the alignment of the overlays seemed to be good (Fig. 13). The feedback from the surgeon was positive regarding the visualization of structures and targets, but he found that the position of the tracker put excessive restrictions on the movements when using tracked tools, so he proposed a change in the position of the tracker for the next tests.
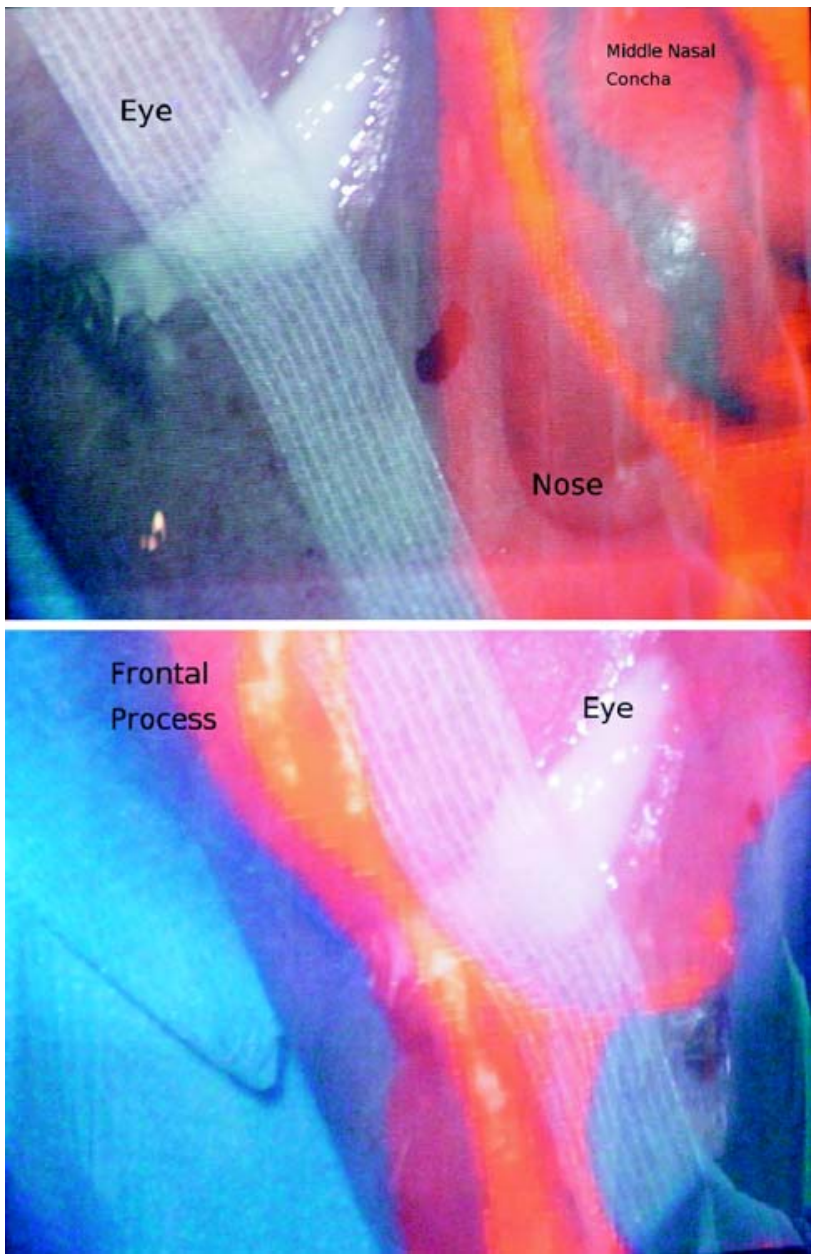

Fig. 13 Planes from the CT Volume are displayed in the microscope view (red color). Several anatomical structures can be identified

The third patient was affected of a malignant tumor, an adenocarcinoma of the nose. In addition to the CT views and the 3D model of the target (Fig. 14), we planned to display the risk regions that were close to the region of interest, like the eye or the closest part of the brain. The registration of the patient was performed in this case with $0.7 \mathrm{~mm}$ accuracy. The new position of the tracker increased the working volume, so the surgeon was able to better use the tracked tools. The system displayed the segmented structures and the surgeon was able to work with the virtual tools and measure distances and intersections (Fig. 15). The measurements taken with the tools were useful in order to see how close the surgeon was to the risk regions, and to point the tools following a defined trajectory to intercept the targets.

Although it is difficult to calculate the accuracy of an augmented reality system on an in vivo case, we managed to take several values with the help of the tracked 


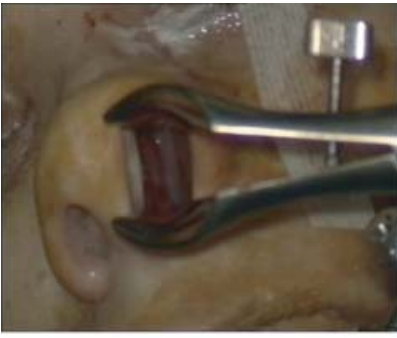

(a) Microscope view of the patient.

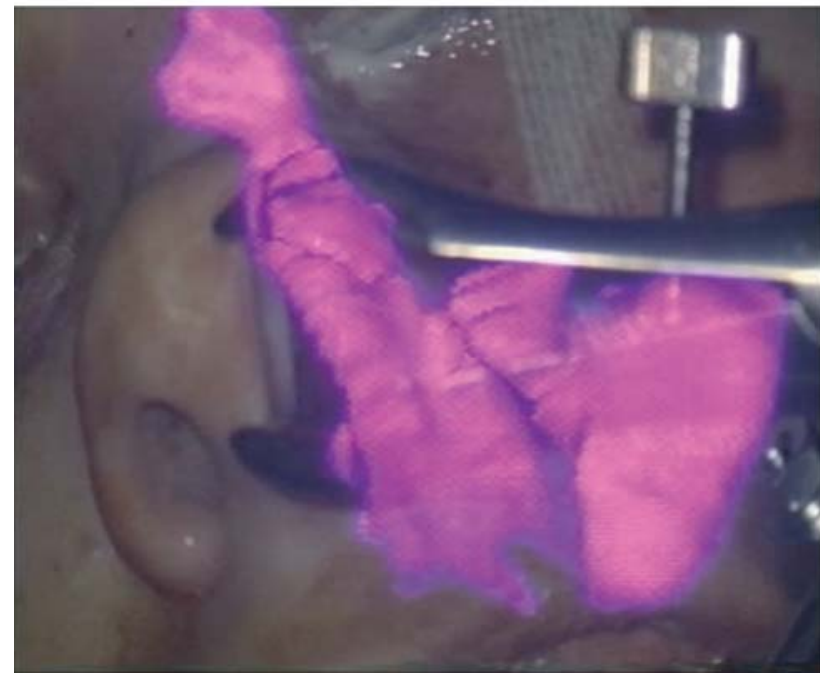

Fig. 14 The 3D model of the malignant tumor segmented from the CT dataset of the patient is rendered and displayed directly to the microscope view

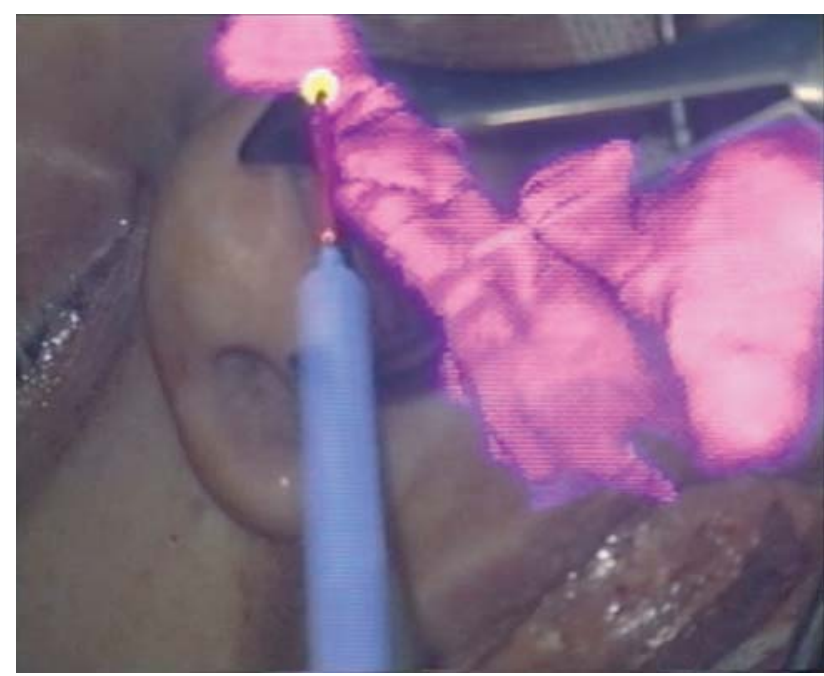

Fig. 15 Interactivity with the augmented reality system. A virtual model of the tool is overlayed to the real tool. Trajectory (red) and intersections (yellow) are displayed

tools by touching segmented structures and measuring distances. Moreover several images were captured in order to post process them and calculate the accuracy using the contours. The result was an error from 1 to $2 \mathrm{~mm}$, with which the surgeon visually agreed.

\section{Discussion}

A new augmented reality system for surgical microscopy has been developed in order to provide guidance to the surgeon during the operation. Since the target of such a surgical microscope is located in the area of head and neck, a high accuracy of the overlay system is essential. Such accuracy has been reached, as proved by our validation studies.

The main novelty of our augmented reality system is the integration of the tracking camera into the microscope. This setup offers improved accuracy since we only need to track the patient and tools, and not the microscope, which is rigidly fixed to the camera. An accurate calibration of the fixation has been developed, together with a semi-automatic calibration of the microscope optics. Moreover the error from fixing the tracker could be reduced with the integration of the camera in a final product. In addition, the miniaturization and integration of such a tracking camera offer improved ergonomics since it is much easier for the surgeon to keep an unobstructed line of sight to tracked objects, and we do not occupy additional space in the operation room.

Accurate patient registration is obtained by fixing a marker shield with a dental splint. Pair-point and surface matching algorithms are then applied by digitizing several anatomical landmarks, with a final accuracy of $0.6 \mathrm{~mm}$. In order to be less invasive and reduce registration time, we avoid the incorporation of bone-implanted fiducials, being the accuracy obtained good enough to perform the experiments.

The system integrates basic navigation techniques like a CT viewer with different angles and tracked pointers, together with a more complex viewer including 3D rendering and virtual 3D tools. Such tools allow the surgeon to interact with the system by measuring distances, trajectories and intersections. Moreover, these measurements provide the surgeon with an increased depth perception and an easier orientation when finding targets.

A complete accuracy study has been done to validate the system prior to clinical application. Several ground truth objects have been used in this study in order to have a complete range of measurements, e.g. grids and phantom skulls. Moreover, registration and visualization have been tested with cadavers to have a more realistic environment. Our results show an accuracy of sub-millimeter magnitude.

Our first clinical cases were successful, and the surgeon validated the accuracy of the system. This is part of a larger clinical study that is in progress. More clinical 
tests are planned in order to evaluate the accuracy of the system, as well as to improve the visualization and interactivity. Discussions with the surgeons are determinant to improve our augmented reality system. Finally, the system will be focused on several applications where specific planning tools will be developed.

Acknowledgments This work was funded by the Swiss National Center of Competence in Research on Computer Aided and Image Guided Medical Interventions (CO-ME), and the Swiss Innovation Promotion Agency KTI/CTI project no. 6997.2. The authors wish to thank the Platform team at MEM Center for their valuable contributions to several modules described in this paper, as well as the staff of Atracsys LLC for their valuable support.

\section{References}

1. Aschke M, Wirtz C, Raczkowsky J, Worn H, Kunze S (2003) Augmented reality in operating microscopes for neurosurgical interventions. In: Proceedings of CAS-H international symposium on computer aided surgery around the head

2. Baechler R, Bunke H, Nolte LP (2001) Restricted surface matching - numerical optimization and technical evaluation. Comput Aided Surg 6(3):143-152

3. Birkfellner W, Figl M, Huber K, Watzinger F, Wanschitz F, Hummel J, Hanel R, Greimel W, Homolka P, Ewers R, Bergmann H (2002) A head-mounted operating binocular for augmented reality visualization in medicine-design and initial evaluation. IEEE Trans on Med Imaging 21(8):991-997

4. Brinker T, Arango G, Kaminsky J, Samii A, Thorns U, Vorkapic P, Samii M (1998) An experimental approach to image guided skull base surgery employing a microscopebased neuronavigation system. Acta Neurochir 140(9):883889

5. Edwards P, King A, Maurer C, Cunha D, Hawkes D, Hill D, Gaston R, Fenlon M, Jusczyzck A, Strong A, Chandler C, Gleeson M (2000) Design and evaluation of a system for microscope-assisted guided interventions (magi). IEEE Transactions on Medical Imaging 19:1082-1093

6. Fichtinger G, Deguet A, Masumane K, Balogh E, Fischer G, Matthieu H, Taylor RH, Zinreich S, Fayad L (2005) Image overlay guidance for needle insertion in ct scanner. IEEE Trans Biomed Eng 52(8):1415-1424

7. Fitzpatrick West J, Maurer C (1998) Predicting error in rigid-body point-based registration. IEEE Trans Med Imag 17(5):694-702
8. Grimson W, Ettinger G, White S, Lozano-Perez T, Wells W, Kikinis R (1996) An automatic registration method for frameless stereotaxy, image guided surgery, and enhanced reality visualization. IEEE Trans Med Imaging 15(2):129-140

9. Hata N, William M, Halle M, Nakajima S, Viola P, Kikinis R, Jolesz F (1996) Image guided microscopic surgery system using mutual-information based registration. In: 4th international conference on visualization in biomedical computing, pp 307-316

10. Heikkila J, Silven O (1997) A four-step camera calibration procedure with implicit image correction. In: IEEE computer society conference on computer vision and pattern recognition, pp 1106-1112

11. Khamene A, Wacker F, Vogt S, Azar F, Wendt M, Sauer F, Lewin J (2003) An augmented reality system for mri-guided needle biopsies. In: Proceedings of medicine meets virtual reality, pp 151-157

12. Kiya N, Dureza C, Fukushima T, Maroon J (1997) Computer navigational microscope for minimally invasive neurosurgery. J Minim Invasive Neurosurg 40(3):110-115

13. Liao H, Hata N, Nakajima S, Iwahara M, Sakuma I, Dohi T (2004) Surgical navigation by autostereoscopic image overlay of integral videography. IEEE Trans Inform Technol Biomed 8(2):114-121

14. Maurer C, Fitzpatrick M, Wang M, Galloway R, Maciunas R, Allen G (1997) Registration of head volume images using implantable fiducial markers. IEEE Trans Med Imaging 16:447-461

15. Salb T, Brief J, Burgert O, Hassfeld S, Mühling J, Dillmann R (1999) An augmented reality system for intraoperative presentation of planning and simulation results. In: Proceedings of workshop: European advanced robotic systems develpment - medical robotics (EUREL), Pisa

16. Schnaider M, Schwald B, Seibert H, Weller T (2003) Medarpa - a medical augmented reality system for minimal-invasive interventions. In: Proceedings of the 11th annual medicine meets virtual reality (MMVR) conference

17. Tsai R (1986) An efficient and accurate camera calibration technique for 3D machine vision. In: IEEE conference on computer vision and pattern recognition, Miami Beach, pp 364-374

18. Veldpaus F, Woltring H, Dortmans L (1988) A least-squares algorithm for the equiform transformation from spatial marker co-ordinates. J Biomech 21:45-54

19. Weber S, Lüth T (2004) A new navigated image viewer with an integrated position sensor. CAS-H

20. Zhang Z (1999) Flexible camera calibration by viewing a plane from unknown orientations. In: International conference on computer vision (ICCV'99), Corfu 